\title{
Asymptotic End-to-end Backlog Evaluation in a Packet Network with a Wide Range of Traffic Flows Including Fractional Brownian Motions
}

\author{
Kazutomo Kobayashi \\ Graduate School of Engineering \\ Nagasaki University \\ Nagasaki 852-8521, Japan \\ kobayashi@cis.nagasaki-u.ac.jp
}

\author{
Yukio Takahashi \\ Tokyo Institute of Technology \\ Tokyo 152-8552, Japan \\ yukio@is.titech.ac.jp
}

\begin{abstract}
The purpose of this paper is to provide a simple evaluation formula for the asymptotic tail probability of the end-to-end backlog in a packet network with a wide range of traffic flows including fractional Brownian motions (fBms).

In the previous paper, the authors proposed the concept of tractable effective bandwidth (tEBW). It is the effective bandwidths (EBWs) of one type and can carry out the endto-end evaluation into a single node evaluation at the bottleneck node. Though almost all of the known traffic flow models have tEBWs, but fBms don't.

In this paper we discuss the end-to-end evaluation under mixed traffic flows with tEBWs and $\mathrm{fBm}$ variances to include fBms. We show that, by suitably evaluating the input traffics (both forwarding traffic and the cross traffics), the end-to-end evaluation can be reduced into a single node evaluation, and obtain a simple asymptotic evaluation formula. The formula depends on the evaluation of the input traffics, but it is independent of the number of nodes as the case for tEBWs only.
\end{abstract}

\section{Categories and Subject Descriptors}

G.3 [Probability and statistics]: Queueing theory; C.2 [Computer communication networks]: Miscellaneous; B.8.2 [Performance and reliability]: Performance analysis and design aids

\section{Keywords}

End-to-end performance evaluation, Effective bandwidth, Fractional Brownian motion, Stochastic network calculus

\section{INTRODUCTION}

This paper proposes a simple evaluation formula for the asymptotic tail probability of the end-to-end backlog in a tandem network with cross traffics in which input traffics are consisting of both fractional Brownian motions (fBms) and flows with tractable effective bandwidths (tEBWs), or mixtures of them. The evaluation formula is an extension of those obtained in previous papers by the authors for models with more restricted input traffics $[7,9]$.

In [9], the authors discussed a heterogeneous tandem network with many forwarding flows and cross traffic flows constrained by leaky buckets, and using the stochastic network calculus for many flows [8] they provided a simple end-to-end evaluation formula for admission control. By the formula, the tail probability of the end-to-end backlog is evaluated from the stochastic characteristics of the total traffic load at a bottleneck node, and hence the evaluation is independent of the number of nodes.

In [7], the authors extend the simple end-to-end evaluation formula for constrained flows with leaky buckets to that for generalized traffic flows with the cumulant generating function $\mathcal{A}(\theta ; t)=\log E\left[e^{\theta A(t)}\right]$ of the arrivals $A(t)$ bounded from above as

$$
\mathcal{A}(\theta ; t) \leq \theta t \cdot \phi(\theta)
$$

where $\phi(\theta)$ is a continuous and strictly increasing function of $\theta$ but independent of time $t$.

Using the effective bandwidth (EBW) $a(\theta, t)$ of $A(t)$ [6], we replace $(1)$ as $a(\theta, t) \leq \phi(\theta)$. Then we call $\phi(\theta)$ as a tractable effective bandwidths, shortly a tEBW, since it is so tractable that the evaluation formula using the tEBW is very simple and convenient. In fact, the end-to-end back log evaluation formula for a tandem network with cross traffic is given as

$$
\limsup _{L \rightarrow \infty} L^{-1} \log P\left(Q^{L}(t)>L b\right) \leq-\theta^{*} b,
$$

where $\theta^{*}$ is the solution of $\phi_{0}(\theta)=c_{0}$. Here, $L, Q^{L}(t)$ and $b$ are the number of forwarding flows, the end-to-end backlog at time $t$, and the buffer threshold per forwarding flow, respectively, and $\phi_{0}(\theta)$ and $c_{0}$ are the total of tEBWs and the link capacity per forwarding flow at a bottleneck node, respectively.

If an EBW is bounded from above by a tEBW, we say it has a tEBW, or the flow has a tEBW. In [7], the authors check each of EBWs discussed in [6] whether it has a tEBW or not. The results show that fractional Brown- 


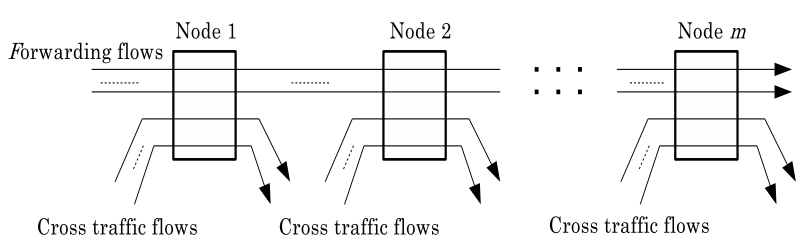

Figure 1: Tandem network with cross traffic

ian motion (fBm) flows with long-range dependency (see, e.g., [13]) don't have any tEBW while all other flows have. The cumulant generating function of the $\mathrm{fBm}$ flow is given as

$$
\mathcal{A}(\theta ; t)=\theta t \cdot \lambda+\frac{1}{2} \sigma^{2} \theta^{2} t^{2 H}
$$

where $\lambda, H$ and $\sigma^{2} t^{2 H}$ are the mean rate of $A(t)$, the Hurst parameter taking a value in the interval $(0.5,1)$ and the variance of $A(t)$, respectively. The asymptotic evaluation formula for a single node with $\mathrm{fBm}$ traffic is well-known [4, $6,13]$.

In this paper, we discuss the end-to-end evaluation under the assumption that the traffic flows satisfy the following type of conditions

$$
\mathcal{A}(\theta ; t) \leq \theta t \cdot \phi(\theta)+\frac{1}{2} \sigma^{2} \theta^{2} t^{2 H},
$$

where $\phi(\theta)$ is a non-decreasing function. This is a condition for a mixed flow of ones having tEBWs and fBms. We show that the asymptotic tail probability of the end-to-end backlog can be evaluated by the backlog analysis of an imaginary single node model with an input traffic consisting of flows satisfying a condition of type (4) and a constant service rate. The resultant evaluation formula does not depend on the number of nodes.

There are many related works [1-14], but most of them treat network models with traffic flows having tEBWs only or with fBms traffic only. In [14], which is highly-related to the issue of this paper, end-to-end performance bounds are obtained for networks with fBm cross traffic in the stochastic network calculus. The forward traffic used is the $\mathrm{fBm}$, EBB (exponential bounded burstiness) or CBR (constant bit rate). By suitably extending their discussion, one might be able to obtain bounds for networks with mixed flows of $\mathrm{fBms}$ and non-fBm processes as treated in this paper. However, their bounds depend on the number of nodes $m$. In this paper, we take an asymptotic approach, and obtain a bound that is independent of $m$. In this sense, our discussion and results are much different from those in [14].

The remainder of the paper is constructed as follows. In Section 2, we describe our network model. In Section 3, we first refer a lemma proved in [7]. It is our starting point of deriving a simple evaluation formula. Then we give flow characterization, and state our main result, Theorem 1 . The proof of the theorem is given in Section 5. In Section 4, some numerical results are presented.

\section{MODEL}

We consider an $m$-node tandem network with cross traffic depicted in Figure 1. Time $t$ takes discrete values $0,1,2, \ldots$ Each of the forwarding traffic and the cross traffic is a set of traffic flows, where a flow is a minimum unit of traffic. The forwarding traffic consists of $L$ flows ${ }^{1}$ and the cross traffic at node $i$ consists of $L_{i}\left(=\gamma_{i} L\right)$ flows, $i=1,2, \ldots, m$. Further specification of flows will be given in Section 3.2.

For time $t \geq 0$, let $A^{L}(t)$ and $A_{i}^{L, \text { cross }}(t)$ be random variables representing the total arrivals of the forwarding traffic for $L$ flows and the total arrivals of the cross traffic at node $i$ for $L_{i}$ flows, respectively, during time interval $(0, t]$, with a convention $A^{L}(0)=A_{i}^{L \text {,cross }}(0)=0$.

For latter use we introduce some bivariate functions. For any pair of times $s$ and $t$ such that $0 \leq s \leq t$, we let

$$
\begin{aligned}
& \bar{A}^{L}(s, t)=A^{L}(t)-A^{L}(s), \quad \text { and } \\
& \bar{A}_{i}^{L, \text { cross }}(s, t)=A_{i}^{L, \text { cross }}(t)-A_{i}^{L, \text { cross }}(s) .
\end{aligned}
$$

The link capacity at node $i$ is constant in time and equal to $L c_{i}$, that is, $c_{i}$ is the link capacity per forwarding flow at node $i$. Regarding the scheduling at each node, we assume that the total offered services $\bar{S}_{i}^{L}(s, t)$ for the forwarding traffic at node $i$ during $(s, t]$ is given by the "leftover" bandwidth

$$
\bar{S}_{i}^{L}(s, t)=\left[L c_{i}(t-s)-\bar{A}_{i}^{L, \operatorname{cross}}(s, t)\right]^{+}
$$

where $[X]^{+}=\max \{0, X\}$. Since generally $\bar{S}_{i}^{L}(s, t) \neq \bar{S}_{i}^{L}(0, t)-$ $\bar{S}_{i}^{L}(0, s)$, it is difficult to discuss the scheduling based on the total offered services $S_{i}^{L}(t)$ during $(0, t]$.

Let $Q^{L}(t)$ be the total backlog of $L$ flows of the forwarding traffic in the whole network at time $t$. With the convolution and deconvolution operators in the network calculus, it can be represented as

$$
Q^{L}(t)=\bar{A}^{L} \oslash\left(\bar{S}_{1}^{L} * \bar{S}_{2}^{L} * \cdots * \bar{S}_{m}^{L}\right)(t, t)
$$

with probability one (see $[1,5,8])$. Here the convolution operator $*$ and the deconvolution operator $\oslash$ are defined as

$$
\begin{aligned}
& f * g(s, t)=\min _{s \leq \tau \leq t}\{f(s, \tau)+g(\tau, t)\}, \quad \text { and } \\
& f \oslash g(s, t)=\max _{0 \leq \tau \leq s}\{f(\tau, t)-g(\tau, s)\},
\end{aligned}
$$

for functions $f(s, t)$ and $g(s, t)$ of two variables $s$ and $t$ such that $0 \leq s \leq t$.

From the definitions of the convolution and deconvolution operators, we easily see that, using a normal maximization operation, it is rewritten as

$$
\begin{aligned}
& Q^{L}(t)=\max _{0 \leq s_{0} \leq \cdots \leq s_{m}=t}\{ \\
& \left.\bar{A}^{L}\left(s_{0}, s_{m}\right)-\bar{S}^{L}\left(s_{0}, s_{1}\right)-\cdots-\bar{S}^{L}\left(s_{m-1}, s_{m}\right)\right\},
\end{aligned}
$$

where the maximization is taken over all possible choices of integers $s_{0}, s_{1}, \cdots, s_{m}$ satisfying

$$
0 \leq s_{0} \leq s_{1} \leq \cdots \leq s_{m-1} \leq s_{m}=t .
$$

\section{EVALUATION OF THE TAIL PROBABILITY OF THE BACKLOG}

\subsection{Cumulant generating function and funda- mental lemma}

In order to evaluate the tail probability of $Q^{L}(t)$, we use cumulant generating functions (cgfs) and asymptotic cumulant generating functions (asymptotic cgfs). For a random

${ }^{1}$ Later, we consider the asymptotic situation where $L$ tends to infinity. 
variable $X^{L}$, its cumulant generating function (cgf) is defined by

$$
\mathcal{X}^{L}(\theta)=\log \mathrm{E}\left[e^{\theta X^{L}}\right], \quad \theta \in \mathbb{R} .
$$

For a sequence of random variables $\left\{X^{L}\right\}_{L=1,2, \ldots}$, its asymptotic cumulant generating function (asymptotic cgf) is defined by

$$
\mathcal{X}(\theta)=\limsup _{L \rightarrow \infty} L^{-1} \mathcal{X}^{L}(\theta), \quad \theta \in \mathbb{R} .
$$

We allow $\pm \infty$ for the values of the cgf and the asymptotic cgf. On the treatment of $\pm \infty$ in mathematical operations we follow the usual convention. Note that if $X^{L}$ is nonnegative with probability one, $\mathcal{X}^{L}(\theta)$ and $\mathcal{X}(\theta)$ are nonnegative for $\theta>0$ and nonpositive for $\theta<0$.

We denote the asymptotic cgf of $\left\{\bar{A}^{L}(s, t)\right\}_{L=1,2, \ldots}$ as $\overline{\mathcal{A}}(\theta ; s, t)$, and that of $\left\{\bar{A}_{i}^{L, \text { cross }}(s, t)\right\}_{L=1,2, \ldots}$ as $\overline{\mathcal{A}}_{i}^{\mathrm{cross}}(\theta ; s, t)$

Our starting point of deriving a new evaluation formula for the backlog is Lemma 1 given below. Before stating the lemma, we make some assumptions.

A1. For any positive integers $t, s_{0}, s_{1}, \cdots, s_{m}$ satisfying (12), random variables $\bar{A}^{L}\left(s_{0}, t\right), \bar{A}_{1}^{L, \text { cross }}\left(s_{0}, s_{1}\right), \cdots$, $\bar{A}_{m}^{L, \text { cross }}\left(s_{m-1}, s_{m}\right)$ are mutually independent.

A2. For any $t>0$, there exists $\delta_{\mathrm{A}}(t)>0$, allowing $\delta_{\mathrm{A}}(t)=$ $+\infty$, such that $\overline{\mathcal{A}}(\theta ; 0, t)$ is finite for $\theta<\delta_{\mathrm{A}}(t)$.

Then we have the following lemma (Equation (20) of [7]).

Lemma 1. In the tandem network with cross traffic, under the assumptions A1 and A2, the tail probability of the backlog $Q^{L}(t)$ can be evaluated for any $t>0$ and $b>0$ as

$$
\begin{aligned}
\limsup _{L \rightarrow \infty} L^{-1} \log \mathrm{P}\left(Q^{L}(t)>L b\right) & \\
\leq & \max _{0 \leq s_{0} \leq \cdots \leq s_{m}=t} \inf _{\theta \in\left(0, \delta_{A}(t)\right)}\left\{-\theta b+\overline{\mathcal{A}}\left(\theta ; s_{0}, s_{m}\right)\right. \\
& \left.-\sum_{i=1}^{m}\left[c_{i} \theta\left(s_{i}-s_{i-1}\right)-\overline{\mathcal{A}}_{i}^{\text {cross }}\left(\theta ; s_{i-1}, s_{i}\right)\right]^{+}\right\},
\end{aligned}
$$

where $[X]^{+}=\max \{0, X\}$.

\subsection{Flow characterization}

We assume that all flows (flows in the forwarding traffic and flows in the cross traffics) are categorized into $J$ types. The types of flows are labeled as $j=1,2, \ldots, J$. Let the number of forwarding flows of type $j$ be $L \alpha_{j}$ and the number of cross traffic flows of type $j$ at node $i$ be $L \beta_{i j}$. If there exist no forwarding flows of type $j$ or no cross traffic flows of type $j$ at node $i$, then we consider $\alpha_{j}=0$ or $\beta_{i j}=0$. However, for brevity of discussion, without loss of generality, we assume $\alpha_{j}+\sum_{i=1}^{m} \beta_{i j}>0$ for every $j$. The total number of forwarding flows is equal to $L \sum_{j=1}^{J} \alpha_{j}$, and hence $\sum_{j=1}^{J} \alpha_{j}=1$. The total number of cross traffic flows at node $i$ is given by $L \sum_{j=1}^{J} \beta_{i j}$, and hence $\sum_{j=1}^{J} \beta_{i j}=\gamma_{i}$. When we move $L$ later, $\alpha_{j}$ 's, $\beta_{i j}$ 's, and $c_{i}$ 's are kept constant.

For individual flows, we make the following assumptions:
C1. All flows are mutually independent.

C2. Each flow has stationary increments.

C3. Flows of type $j$ are subjecting to a common probabilistic law.

C4. Let $A_{j}(t)$ be the random variable representing the total arrivals to the network during time interval $(0, t]$ of a typical flow of type $j$, with a convention $A_{j}(0)=0$. The cgf $\mathcal{A}_{j}(\theta ; t)=\log E\left[e^{\theta A_{j}(t)}\right]$ of $A_{j}(t)$ is bounded from above as $^{2}$

$$
\mathcal{A}_{j}(\theta ; t) \leq \theta t \cdot \phi_{j}(\theta)+\frac{1}{2} \sigma_{j}^{2} \theta^{2} t^{2 H_{j}}
$$

for $\theta \in[0, \infty)$, where $\sigma_{j}$ is a parameter related to the variance of $A_{j}(t), H_{j}$ is the Hurst parameter taking a value in the interval $(0.5,1)$, and $\phi_{j}(\theta)$ is a function defined on $[0, \infty)$ such that it is non-decreasing on $\left[0, \delta_{j}\right)$ for some $\delta_{j}>0$ (including the case $\delta_{j}=\infty$ ) and $\phi_{j}(\theta)=\infty$ for $\theta>\delta_{j}$. We define

$$
\delta_{0}=\min _{j \in\{1,2, \cdots, m\}}\left\{\delta_{j}\right\} .
$$

If all $\delta_{j}$ 's are infinity, then $\delta_{0}$ is infinity.

From the assumptions $\mathrm{C} 2$ and $\mathrm{C} 4$, the $\operatorname{cgf} \overline{\mathcal{A}}_{j}(\theta ; s, t)$ of $\bar{A}_{j}(s, t)=A_{j}(t)-A_{j}(s), 0 \leq s \leq t$, can be evaluated as

$$
\overline{\mathcal{A}}_{j}(\theta ; s, t) \leq \theta(t-s) \phi_{j}(\theta)+\frac{1}{2} \theta^{2}(t-s)^{2 H_{j}} \sigma_{j}^{2} .
$$

Denoting the $k$ th flow of type $j$ of the forwarding traffic as $A_{j, k}(t)$, the increment of arrivals in the forwarding traffic during $(s, t]$ is written as

$$
\bar{A}^{L}(s, t)=\sum_{j=1}^{J} \sum_{k=1}^{L \alpha_{j}}\left\{A_{j, k}(t)-A_{j, k}(s)\right\},
$$

and from the assumptions $\mathrm{C} 1$ and $\mathrm{C} 3$, its cgf is given by

$$
\sum_{j=1}^{J} L \alpha_{j} \overline{\mathcal{A}}_{j}(\theta ; s, t)
$$

Since $\overline{\mathcal{A}}_{j}(\theta ; s, t)$ is evaluated as in (18), the asymptotic cgf of the increment of the forwarding flows is evaluated as

$$
\begin{aligned}
\overline{\mathcal{A}}(\theta ; s, t) & \leq \theta(t-s) \sum_{j=1}^{J} \alpha_{j} \phi_{j}(\theta)+\frac{1}{2} \theta^{2} \sum_{j=1}^{J} \alpha_{j} \sigma_{j}^{2}(t-s)^{2 H_{j}} \\
& =\theta(t-s) \phi_{\alpha}(\theta)+\frac{1}{2} \theta^{2} \xi_{\alpha}(t-s)
\end{aligned}
$$

where $\phi_{\alpha}(\theta)$ is a function given by

$$
\phi_{\alpha}(\theta)=\sum_{j=1}^{J} \alpha_{j} \phi_{j}(\theta),
$$

${ }^{2}$ As stated in Section 1, it is said in [7] that the flow $A_{j}(t)$ has a tractable effective band width (tEBW) $\phi_{j}(\theta)$ if

$$
\mathcal{A}_{j}(\theta ; t) \leq \theta t \cdot \phi_{j}(\theta)
$$

for any choice of positive $t$ and $\theta$. On the other hand if $A_{j}(t)$ is a fractional Brownian motion with Hurst parameter $H_{j}$, its cgf is given by

$$
\mathcal{A}_{j}(\theta ; t)=\theta t \cdot \lambda_{j}+\frac{1}{2} \sigma_{j}^{2} \theta^{2} t^{2 H_{j}},
$$

where $\lambda_{j}$ is the mean arrival rate and $\sigma_{j}^{2}$ the variance parameter. The condition (16) is derived by combining these two cases. 
and $\xi_{\alpha}(t)$ is a function of discrete variable $t$ given by

$$
\xi_{\alpha}(t)=\sum_{j=1}^{J} \alpha_{j} \sigma_{j}^{2} t^{2 H_{j}} .
$$

Similarly, denoting the $k$ th flow of type $j$ of the cross traffic at node $i$ as $A_{i, j, k}^{\text {cross }}(t)$, the increment of arrivals in the cross traffic at node $i$ during $(s, t]$ is written as

$$
\bar{A}_{i}^{L, \operatorname{cross}}(s, t)=\sum_{j=1}^{J} \sum_{k=1}^{L \beta_{i j}}\left\{A_{i, j, k}^{\mathrm{cross}}(t)-A_{i, j, k}^{\mathrm{cross}}(s)\right\},
$$

and from the assumptions $\mathrm{C} 1$ and $\mathrm{C} 3$, its cgf is given by

$$
\sum_{j=1}^{J} L \beta_{i j} \overline{\mathcal{A}}_{j}(\theta ; s, t) .
$$

Then the asymptotic cgf of the increment of the cross traffic at node $i$ is evaluated as

$$
\begin{aligned}
& \overline{\mathcal{A}}_{i}^{\text {cross }}(\theta ; s, t) \\
& \leq \theta(t-s) \sum_{j=1}^{J} \beta_{i j} \phi_{j}(\theta)+\frac{1}{2} \theta^{2} \sum_{j=1}^{J} \beta_{i j} \sigma_{j}^{2}(t-s)^{2 H_{j}} \\
& =\theta(t-s) \phi_{\beta, i}(\theta)+\frac{1}{2} \theta^{2} \xi_{\beta, i}(t-s)
\end{aligned}
$$

where $\phi_{\beta, i}(\theta)$ is a function given by

$$
\phi_{\beta, i}(\theta)=\sum_{j=1}^{J} \beta_{i j} \phi_{j}(\theta),
$$

and $\xi_{\beta, i}(t)$ is a function of discrete variable $t$ given by

$$
\xi_{\beta, i}(t)=\sum_{j=1}^{J} \beta_{i j} \sigma_{j}^{2} t^{2 H_{j}} .
$$

Then from (26), we have

$$
\begin{aligned}
& {\left[c_{i} \theta\left(s_{i}-s_{i-1}\right)-\overline{\mathcal{A}}_{i}^{\mathrm{cross}}\left(\theta ; s_{i-1}, s_{i}\right)\right]^{+}} \\
& \geq\left[\theta\left(s_{i}-s_{i-1}\right)\left(c_{i}-\phi_{\beta, i}(\theta)\right)-\frac{1}{2} \theta^{2} \xi_{\beta, i}\left(s_{i}-s_{i-1}\right)\right]^{+}
\end{aligned}
$$

Applying (21) and (29) to (15), we obtain the following lemma straightforwardly. Note that the assumptions A1 and A2 are automatically satisfied from the assumptions $\mathrm{C} 1$ to C4.

Lemma 2. For a given time $t>0$ and a given $b>0$, under the assumptions $\mathrm{C} 1$ to $\mathrm{C} 4$, we have

$$
\begin{gathered}
\limsup _{L \rightarrow \infty} L^{-1} \log \mathrm{P}\left(Q^{L}(t)>L b\right) \\
\leq \max _{0 \leq s_{0} \leq \cdots \leq s_{m}=t} \inf _{\theta \in\left(0, \delta_{0}(t)\right)}\{ \\
-\theta b+\theta\left(s_{m}-s_{0}\right) \phi_{\alpha}(\theta)+\frac{1}{2} \theta^{2} \xi_{\alpha}\left(s_{m}-s_{0}\right) \\
-\sum_{i=1}^{m}\left[\theta\left(s_{i}-s_{i-1}\right)\left(c_{i}-\phi_{\beta, i}(\theta)\right)\right. \\
\left.\left.-\frac{1}{2} \theta^{2} \xi_{\beta, i}\left(s_{i}-s_{i-1}\right)\right]^{+}\right\} .
\end{gathered}
$$

\subsection{Simple evaluation formula}

The evaluation formula (30) is very complicated. To derive a more simple and tractable formula, we make further some technical assumptions ${ }^{3}$.

C5. The stability condition

$$
\phi_{\alpha}(0)+\phi_{\beta, i}(0)<c_{i}, i=1,2, \cdots, m .
$$

hold for $\theta \in[0, \infty)$.

C6. There exist a positive constant $c_{0}$ and a continuously twice differentiable, non-decreasing, convex function $\phi_{0}(\theta)$ defined on $\left[0, \delta_{0}\right)$ such that

$$
\begin{gathered}
c_{i}-\phi_{\alpha}(\theta)-\phi_{\beta, i}(\theta) \geq c_{0}-\phi_{0}(\theta), \\
\quad \text { for } \theta \in\left[0, \delta_{0}\right), i=1,2, \cdots, m, \\
\phi_{0}(0)<c_{0}, \quad \text { and } \quad \lim _{\theta \uparrow \delta_{0}} \phi_{0}(\theta)=\infty .
\end{gathered}
$$

C7. (i) There exists at least one $j$ such that $\sigma_{j}^{2}>0$.

(ii) The time $t$ is large enough (see footnote 3 ).

(iii) The function $\left(c_{0}-\phi_{0}(\theta)\right)^{2}$ is convex on $\theta$ in the interval $\left(0, \theta^{\dagger}\right)$, where $\theta^{\dagger}$ is a unique positive value of $\theta$ such that $\phi_{0}(\theta)=c_{0}$.

For the new evaluation, we prepare some notations.

$$
\begin{aligned}
& H_{0}=\max _{j \in\{1,2, \cdots, J\}} H_{j}, \\
& \sigma_{\alpha}^{2}=\sum_{j=1}^{J} \alpha_{j} \sigma_{j}^{2}, \quad \sigma_{\beta, i}^{2}=\sum_{j=1}^{J} \beta_{i j} \sigma_{j}^{2}, \quad i=1,2, \ldots, m, \\
& \sigma_{\beta, 0}^{2}=\max _{i \in\{1,2, \cdots, m\}}\left\{\sigma_{\beta, i}^{2}\right\},
\end{aligned}
$$

and

$$
\sigma_{0}^{2}=\sigma_{\alpha}^{2}+\sigma_{\beta, 0}^{2} .
$$

Then, clearly

$$
\xi_{\alpha}(\tau) \leq \sigma_{\alpha}^{2} \tau^{2 H_{0}} \quad \text { and } \quad \xi_{\beta, i}(\tau) \leq \sigma_{\beta, 0}^{2} \tau^{2 H_{0}}
$$

for $\tau \in\{1,2, \cdots, t\}$ and $i=1,2, \cdots, m$.

We denote the function in the braces of the right-hand side of $(30)$ as $G\left(\theta ; s_{0}, \cdots, s_{m}\right)$. Then it is evaluated as follows. Here we use (i) the inequality $-[x]^{+} \leq-x$ for any $x$, (ii) the inequalities (32) and (36), and (iii) the convexity of $\tau^{2 H_{0}}$.

\footnotetext{
${ }^{3}$ These are technical assumptions to avoid trivial cases or to make the analysis easier. The constant $c_{0}$ in C6 is introduced only for making the both sides of the inequality (32) being of the same form. The value of $c_{0}$ can be arbitrarily set. For example we may put $c_{0}=0$.

The stability condition (31) in C5 is not used later. It is inserted here because the existence of $c_{0}$ and $\phi_{0}(\theta)$ in C6 is not realistic without it.

Assumption (i) in $\mathrm{C} 7$ requests that $\sigma_{0}^{2}$ defined in (35) is positive. Assumption (ii) is imposed so that a solution to the equations (41) in Theorem 1 exists in the interior of the domain of function $F(\theta ; \tau)$. Assumption (iii) assures that the solution is unique.
} 


$$
\begin{aligned}
& G\left(\theta ; s_{0}, \cdots, s_{m}\right) \\
& \leq-\theta b+\theta\left(s_{m}-s_{0}\right) \phi_{\alpha}(\theta)+\frac{1}{2} \theta^{2} \xi_{\alpha}\left(s_{m}-s_{0}\right) \\
&-\sum_{i=1}^{m}\left\{\theta\left(s_{i}-s_{i-1}\right)\left(c_{i}-\phi_{\beta, i}(\theta)\right)-\frac{1}{2} \theta^{2} \xi_{\beta, i}\left(s_{i}-s_{i-1}\right)\right\} \\
&=-\theta b-\theta \sum_{i=1}^{m}\left(s_{i}-s_{i-1}\right)\left(c_{i}-\phi_{\alpha}(\theta)-\phi_{\beta, i}(\theta)\right) \\
&+\frac{1}{2} \theta^{2} \xi_{\alpha}\left(s_{m}-s_{0}\right)+\frac{1}{2} \theta^{2} \sum_{i=1}^{m} \xi_{\beta, i}\left(s_{i}-s_{i-1}\right) \\
& \leq-\theta b-\theta \sum_{i=1}^{m}\left(s_{i}-s_{i-1}\right)\left(c_{0}-\phi_{0}(\theta)\right) \\
&+\frac{1}{2} \theta^{2} \sigma_{\alpha}^{2}\left(t-s_{0}\right)^{2 H_{0}}+\frac{1}{2} \theta^{2} \sigma_{\beta, 0}^{2} \sum_{i=1}^{m}\left(s_{i}-s_{i-1}\right)^{2 H_{0}} \\
& \leq-\theta\left\{b+\left(t-s_{0}\right)\left(c_{0}-\phi_{0}(\theta)\right)\right\}+\frac{1}{2} \theta^{2} \sigma_{0}^{2}\left(t-s_{0}\right)^{2 H_{0}} .
\end{aligned}
$$

Notice that variables $s_{1}, s_{2}, \cdots, s_{m-1}$ don't appear in the last expression of (37). So, if we put

$$
F(\theta ; \tau)=-\theta\left\{b+\tau\left(c_{0}-\phi_{0}(\theta)\right)\right\}+\frac{1}{2} \sigma_{0}^{2} \theta^{2} \tau^{2 H_{0}}
$$

for $\theta \in\left[0, \delta_{0}\right)$ and $\tau=0,1,2, \ldots$, then from (30) and (37) we have an evalutation formula using $F(\theta ; \tau)$ for the tail probability of the end-to-end backlog as

$$
\begin{aligned}
\limsup _{L \rightarrow \infty} L^{-1} \log P\left(Q^{L}(t)>L b\right) \\
\leq \max _{0 \leq s_{0} \leq t} \inf _{\theta \in\left(0, \delta_{0}\right)} F\left(\theta ; t-s_{0}\right) \\
\quad=\max _{\tau \in\{0,1,2, \ldots, t\}} \inf _{\theta \in\left(0, \delta_{0}\right)} F(\theta ; \tau) .
\end{aligned}
$$

For latter convenience, we let the variable $\tau$ in $F(\theta ; \tau)$ be continuous, and $F(\theta ; \tau)$ is a bivariate function of two continuous variables $\theta$ and $\tau$. In other words, we consider that $F(\theta ; \tau)$ in $(38)$ is defined on an enlarged domain $\left[0, \delta_{0}\right) \times$ $[0 . \infty)$. Using (39), we have the following theorem.

Theorem 1. Under the assumptions C1 to C7, for any $b>0$ we have

$$
\limsup _{L \rightarrow \infty} L^{-1} \log P\left(Q^{L}(t)>L b\right) \leq F\left(\theta^{*} ; \tau^{*}\right),
$$

where $\left(\theta^{*}, \tau^{*}\right)$ is a unique solution of the system of equations

$$
\frac{\partial}{\partial \theta} F(\theta ; \tau)=\frac{\partial}{\partial \tau} F(\theta ; \tau)=0,
$$

or more definitely, it is a unique solution of the system of equations

$$
\left\{\begin{array}{l}
-b-\tau\left\{c_{0}-\phi_{0}(\theta)-\theta \phi_{0}^{\prime}(\theta)\right\}+\sigma_{0}^{2} \theta \tau^{2 H_{0}}=0, \\
-\left\{c_{0}-\phi_{0}(\theta)\right\}+H_{0} \sigma_{0}^{2} \theta \tau^{2 H_{0}-1}=0 .
\end{array}\right.
$$

Remark 1. Strictly speaking, the assumption C6 excludes the case $\phi_{0}(\theta)=\lambda_{0}$ (constant) with $\delta_{0}=\infty$ (the case of $f B m$ flows only). However, even in that case, Theorem 1 holds, and the solution to the system of equations (42) is given by

$$
\begin{aligned}
\tau^{*} & =\frac{H_{0} b}{\left(1-H_{0}\right)\left(c_{0}-\lambda_{0}\right)}, \quad \text { and } \\
\theta^{*} & =\frac{\left(1-H_{0}\right)^{2 H_{0}-1}\left(c_{0}-\lambda_{0}\right)^{2 H_{0}}}{H_{0}^{2 H_{0}} \sigma_{0}^{2} b^{2 H_{0}-1}},
\end{aligned}
$$

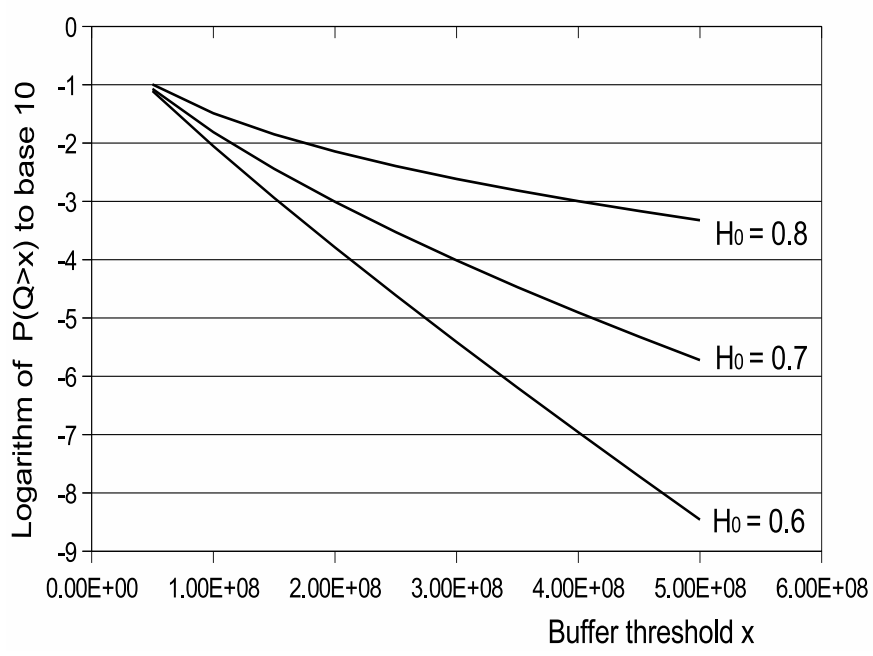

Figure 2: Tail probabilities for $H_{0}=0.6,0.7,0.8$ and $\varrho=2 \mathrm{M}$

and the right hand side of (40) is given by

$$
F\left(\theta^{*} ; \tau^{*}\right)=-\frac{1}{2} \frac{\left(c_{0}-\lambda_{0}\right)^{2 H_{0}} b^{2-2 H_{0}}}{H_{0}^{2 H_{0}}\left(1-H_{0}\right)^{2-2 H_{0}} \sigma_{0}^{2}} .
$$

\section{NUMERICAL EXAMPLES}

We discuss some numerical examples under the situation where $\phi_{0}(\theta), c_{0}, \sigma_{0}^{2}$ and $H_{0}$ are given, namely the function $F(\theta ; \tau)$ of $(38)$ is given. From Theorem 1 we can evaluate the backlog $Q^{L}(t)$ as

$$
P\left(Q^{L}(t)>L b\right) \lesssim e^{L F\left(\theta^{*} ; \tau^{*}\right)} .
$$

Here we use the sign of $\lesssim$ in the sense that $P\left(Q^{L}(t)>L b\right)$ can be approximately evaluated by $e^{L F\left(\theta^{*} ; \tau^{*}\right)}$.

We let

$$
\phi_{0}(\theta)=\frac{\rho}{\theta \varrho}\left(e^{\theta \varrho}-1\right) .
$$

This is a tractable effective bandwidth of flows shaped with leaky buckets with token rate $\rho$ and token bucket size $\varrho[6,7]$. Other parameters are set as $L=50, \rho=9 \mathrm{Mbps}, \varrho=2 \mathrm{Mbits}$, $c_{0}=10 \mathrm{Mbps}$ and $\sigma_{0}^{2}=\rho^{2} / 4$, in common.

Figure 2 shows the results for the mixed flows with Hurst parameter $H_{0}=0.6,0.7$ and 0.8 . We use the bisection method for obtaining the solution of the system of the equations (42). In the figure, the ordinate is $\log _{10} P\left(Q^{L}(t)>x\right)$ and the abscissa is the buffer threshold $x=L b$. We see that the decays show Weibull tails and gets slower as $H_{0}$ gets larger. This feature is the same as in the case of $\mathrm{fBm}$ traffics.

Now we consider the case in which flows have the tEBW with

$$
\theta t \cdot \frac{\rho}{\theta \varrho}\left(e^{\theta \varrho}-1\right)+\frac{1}{2} \sigma_{0}^{2} \theta^{2} t
$$

and the case in which flows have the fBm with

$$
\theta t \cdot \rho+\frac{1}{2} \sigma_{0}^{2} \theta^{2} t^{2 H_{0}}
$$

The former has the same average rate and burstiness as the mixed flows, and the latter has the same average rate and the same Hurst parameter. 


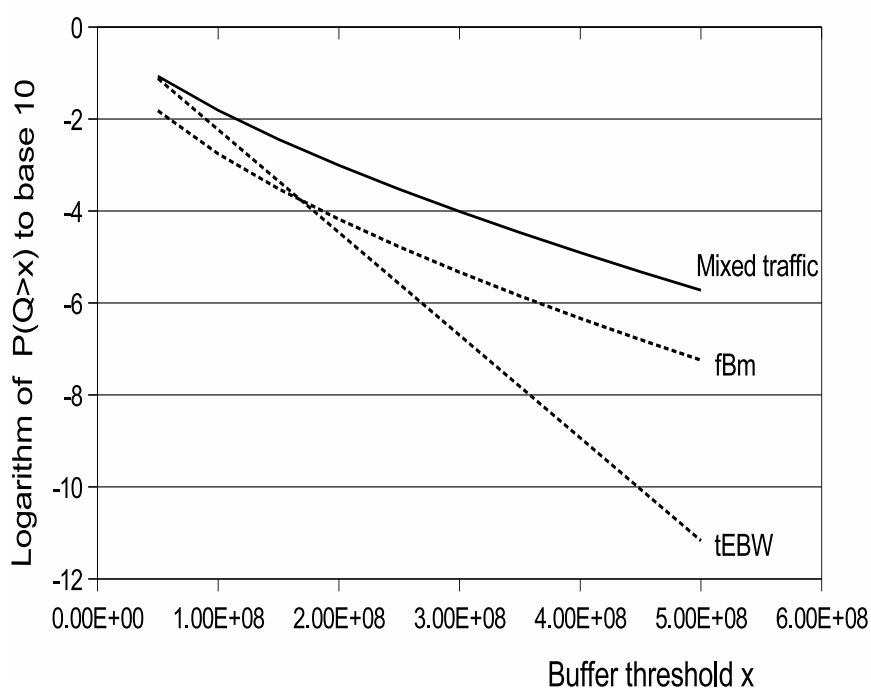

Figure 3: Tail probabilities for mixed flow, tEBW and $\mathrm{fBm}$

Figure 3 shows the results for the mixed flows, the flows with the tEBW and the fBms. The results for the latter cases show some serious underestimates as compared with the result of the mixed flows, because they don't take account of long dependency, $H_{0}=0.7$, and bursty, $\varrho=2 \mathrm{Mbits}$ of the mixed flow, respectively. Especially, the influence of the underestimation is larger for the flows with the tEBW as the buffer threshold $x$ increases. This might indicate that the effect of the long dependency is larger than that of the burstiness.

\section{PROOF OF THEOREM 1}

Theorem 1 is proved through a series of lemmas.

First we shall check that the system of equations (41) is reduced to (42). We denote partial derivatives of $F(\theta ; \tau)$ as $F_{\theta}(\theta ; \tau)=\frac{\partial}{\partial \theta} F(\theta ; \tau)$ and $F_{\tau}(\theta ; \tau)=\frac{\partial}{\partial \tau} F(\theta ; \tau)$. Direct differentiations show that

$$
F_{\theta}(\theta ; \tau)=-b-\tau\left(c_{0}-\phi_{0}(\theta)-\theta \phi_{0}^{\prime}(\theta)\right)+\theta \sigma_{0}^{2} \tau^{2 H_{0}},
$$

and

$$
\begin{aligned}
F_{\tau}(\theta ; \tau) & =-\theta\left(c_{0}-\phi_{0}(\theta)\right)+H_{0} \sigma_{0}^{2} \theta^{2} \tau^{2 H_{0}-1} \\
& =\theta\left[-\left(c_{0}-\phi_{0}(\theta)\right)+H_{0} \sigma_{0}^{2} \theta \tau^{2 H_{0}-1}\right] .
\end{aligned}
$$

Clearly the equation $F_{\theta}(\theta ; \tau)=0$ is equivalent to the first equation of $(42)$. Since $\phi_{0}(0)<c_{0}$ from the assumption C6, any pair $(\theta, \tau)$ with $\theta=0$ cannot satisfy the equation $F_{\theta}(\theta ; \tau)=0$. Hence such a pair is not a solution of (41), and from $(51)$, the equation $F_{\tau}(\theta ; \tau)=0$ reduces to the second equation of (42). Thus the system of equations (42) is equivalent to (41).

Now we proceed to the first lemma for the proof of Theorem 1.

Lemma 3. For a fixed $\tau \in(0, \infty)$, the first equation of (42) on $\theta$ has a unique solution $\theta^{*}(\tau)$. As a function of $\tau$, $\theta^{*}(\tau)$ is monotonically decreasing, and

$$
\lim _{\tau \downarrow 0} \theta^{*}(\tau)=\delta_{0}, \quad \text { and } \quad \lim _{\tau \rightarrow \infty} \theta^{*}(\tau)=0 .
$$

On the convergence when $\tau \rightarrow \infty$, we have a more precise evaluation:

$$
\theta^{*}(\tau)=\frac{c_{0}-\phi_{0}(0)}{\sigma_{0}^{2}} \tau^{1-2 H_{0}}+o\left(\tau^{1-2 H_{0}}\right), \quad \text { as } \tau \rightarrow \infty .
$$

Proof. A solution of the first equation of (42) is given by the $\theta$-coordinate of an intersection of the graphs of the functions

$$
\begin{aligned}
& y=-\frac{b}{\tau}+\sigma_{0}^{2} \theta \tau^{2 H_{0}-1}, \quad \text { and } \\
& y=c_{0}-\phi_{0}(\theta)-\theta \phi_{0}^{\prime}(\theta) .
\end{aligned}
$$

on a $\theta-y$ plane in the region $\left[0, \delta_{0}\right) \times(-\infty, \infty)$. The graph of (54) is a straight line with negative $y$-intercept and positive gradient. On the other hand, the graph of (55) is a curve starting from a positive $y$-intercept and monotonically decreasing to $-\infty$ as $\theta \rightarrow \delta_{0}$. It is clear that these graphs intersects at a single point, and we denote its $\theta$-coordinate by $\theta^{*}(\tau)$. This is the unique solution of (42). As $\tau$ increases, the line moves upward slightly rotating, whereas the curve does not move. Hence we easily see that $\theta^{*}(\tau)$ is monotonically decreasing since both the $y$-intercept and the gradient of the line are increasing on $\tau$. We also easily see from the graphs that the limits are given as in (52).

To see the convergence speed given in (53), we note that, by the Taylor's theorem, (55) can be written as

$$
\begin{aligned}
y= & c_{0}-\phi_{0}(0)-\phi_{0}^{\prime}(0) \theta-\frac{1}{2} \phi_{0}^{\prime \prime}\left(\xi_{1} \theta\right) \theta^{2} \\
& \quad-\theta\left(\phi_{0}^{\prime}(0)+\phi_{0}^{\prime \prime}\left(\xi_{2} \theta\right) \theta\right) \\
= & c_{0}-\phi_{0}(0)-2 \phi_{0}^{\prime}(0) \theta-\left(\frac{1}{2} \phi_{0}^{\prime \prime}\left(\xi_{1} \theta\right)+\phi_{0}^{\prime \prime}\left(\xi_{2} \theta\right)\right) \theta^{2},
\end{aligned}
$$

where $\xi_{1}$ and $\xi_{2}$ are appropriate numbers in the interval $(0,1)$, though they may depend on $\theta$. Since $\phi_{0}^{\prime \prime}(\theta)$ is continuous from the assumption $\mathrm{C} 6$, if we restrict $\theta$ in a finite interval, say in $\left(0, \theta_{0}\right)$, there is a positive number $R$ such that

$$
0 \leq \frac{1}{2} \phi_{0}^{\prime \prime}\left(\xi_{1} \theta\right)+\phi_{0}^{\prime \prime}\left(\xi_{2} \theta\right) \leq R, \quad \theta \in\left(0, \theta_{0}\right) .
$$

Then from (54) and (56), we have

$$
0 \leq B-C \theta \leq R \theta^{2},
$$

where

$$
B=c_{0}-\phi_{0}(0)+b \tau^{-1} \quad \text { and } \quad C=2 \phi_{0}^{\prime}(0)+\sigma_{0}^{2} \tau^{2 H_{0}-1} .
$$

By solving these inequalities on $\theta$, we have

$$
\frac{2 B}{C+\sqrt{C^{2}+4 B R}} \leq \theta \leq \frac{B}{C} .
$$

The unique solution $\theta^{*}(\tau)$ must satisfy these inequalities. When $\tau \rightarrow \infty, B$ tends to $c_{0}-\phi_{0}(0)$ and $C$ diverges to $\infty$, whereas $R$ remains constant. So, we have asymptotic results for the case $\tau \rightarrow \infty$ as follows:

$$
\begin{aligned}
\frac{B}{C}= & \frac{c_{0}-\phi_{0}(0)}{\sigma_{0}^{2}} \tau^{1-2 H_{0}}+\frac{b}{\sigma^{2}} \tau^{-2 H_{0}} \\
& -\frac{c_{0}-\phi_{0}(0)}{\sigma_{0}^{2}} \cdot \frac{2 \phi_{0}^{\prime}(0)}{\sigma_{0}^{2}} \tau^{2-4 H_{0}}+o\left(\tau^{2-4 H_{0}}\right) \\
= & \frac{c_{0}-\phi_{0}(0)}{\sigma_{0}^{2}} \tau^{1-2 H_{0}}+o\left(\tau^{1-2 H_{0}}\right),
\end{aligned}
$$


and

$$
\begin{aligned}
\frac{B}{C} & -\frac{2 B}{C+\sqrt{C^{2}+4 B R}} \\
& =\frac{B}{C} \cdot \frac{4 B R / C^{2}}{\left\{1+\sqrt{1+4 B R / C^{2}}\right\}^{2}} \leq \frac{4 B^{2} R}{C^{3}} \\
& =\frac{4\left(c_{0}-\phi_{0}(0)\right)^{2} R}{\sigma_{0}^{6}} \tau^{3-6 H_{0}}+o\left(\tau^{3-6 H_{0}}\right)=o\left(\tau^{2-4 H_{0}}\right) .
\end{aligned}
$$

Hence

$$
\begin{aligned}
\frac{2 B}{C+\sqrt{C^{2}+4 B R}} & =\frac{B}{C}+o\left(\tau^{2-4 H_{0}}\right) \\
& =\frac{c_{0}-\phi_{0}(0)}{\sigma_{0}^{2}} \tau^{1-2 H_{0}}+o\left(\tau^{1-2 H_{0}}\right),
\end{aligned}
$$

and we see that (53) holds from(57).

Since $\theta^{*}(\tau)$ is a unique solution of (50) and functions $\phi_{0}(\theta)$ and $\phi_{0}^{\prime}(\theta)$ are non-decreasing with $\lim _{\theta \uparrow \delta_{0}} \phi_{0}(\theta)=\infty$, it is clear that $\theta^{*}(\tau)$ is the value of $\theta$ that attains the infimum on $\theta$ in (39). We let

$$
\begin{aligned}
& K(\tau)=F\left(\theta^{*}(\tau) ; \tau\right) \\
& =-\theta^{*}(\tau)\left\{b+\tau\left(c_{0}-\phi_{0}\left(\theta^{*}(\tau)\right)\right)\right\}+\frac{1}{2} \sigma_{0}^{2}\left\{\theta^{*}(\tau)\right\}^{2} \tau^{2 H_{0}}
\end{aligned}
$$

This is the function for which the maximization on $\tau$ in (39) is taken. It is clear that $K(\tau)$ is twice continuously differentiable for $\tau$ in $(0, \infty)$.

Lemma 4. The derivative of $K(\tau)$ is given by

$$
\begin{aligned}
& K^{\prime}(\tau)=\left.F_{\tau}(\theta ; \tau)\right|_{\theta=\theta^{*}(\tau)} \\
& =-\theta^{*}(\tau)\left\{c_{0}-\phi_{0}\left(\theta^{*}(\tau)\right)\right\}+H_{0} \sigma_{0}^{2}\left\{\theta^{*}(\tau)\right\}^{2} \tau^{2 H_{0}-1} .
\end{aligned}
$$

Proof. Since $\left.F_{\theta}(\theta ; \tau)\right|_{\theta=\theta^{*}(\tau)}=0$ from the definition of $\theta^{*}(\tau)$ in Lemma 3 , we have

$$
\begin{aligned}
K^{\prime}(\tau) & =\frac{d}{d \tau} F\left(\theta^{*}(\tau) ; \tau\right) \\
& =\left.F_{\theta}(\theta ; \tau)\right|_{\theta=\theta^{*}(\tau)} \cdot \frac{d \theta^{*}(\tau)}{d \tau}+\left.F_{\tau}(\theta ; \tau)\right|_{\theta=\theta^{*}(\tau)} \\
& =\left.F_{\tau}(\theta ; \tau)\right|_{\theta=\theta^{*}(\tau)} .
\end{aligned}
$$

The second equality in (59) is a direct consequence of (51).

Suppose that there exists a number $\tau^{*}$ such that $K^{\prime}\left(\tau^{*}\right)=$ 0 . Then, the pair $\left(\theta^{*}\left(\tau^{*}\right), \tau^{*}\right)$ is a solution of the system of equations (42), and $\tau^{*}$ is a candidate of $\tau$ at which the maximum of $K(\tau)=F\left(\theta^{*}(\tau) ; \tau\right)$ in (39) is attained. We shall show the existence of $\tau^{*}$ and its uniqueness.

For $\theta^{\dagger}$ defined in the assumption $\mathrm{C} 7$, let $\tau^{\dagger}$ be the value of $\tau$ such that $\theta^{*}(\tau)=\theta^{\dagger}$, or equivalently the value of $\tau$ such that $\phi_{0}\left(\theta^{*}(\tau)\right)=c_{0}$. From the monotonicity of the function $\theta^{*}(\tau)$ shown in Lemma $3, \tau^{\dagger}$ is well defined.

Lemma 5. (i) $K(\tau)$ is increasing on the interval $\left(0, \tau^{\dagger}\right]$. (ii) $\lim _{\tau \rightarrow \infty} K(\tau)=-\infty$.

Proof. (i) Since $\phi_{0}\left(\theta^{*}(\tau)\right)$ is monotonically decreasing, we know from the definition of $\tau^{\dagger}$ that $c_{0}-\phi_{0}\left(\theta^{*}(\tau)\right)<0$ for $\tau \in\left(0, \tau^{\dagger}\right)$. Since $c_{0}-\phi_{0}\left(\theta^{*}\left(\tau^{\dagger}\right)\right)=0$, from (59), we easily see that $K^{\prime}(\tau)>0$ on the interval $\left(0, \tau^{\dagger}\right]$.

(ii) We can rewrite (58) using (42) as

$$
\begin{aligned}
& K(\tau) \\
& =-\frac{1}{2} \theta^{*}(\tau)\left[b+\tau\left\{c_{0}-\phi_{0}\left(\theta^{*}(\tau)\right)+\theta^{*}(\tau) \phi_{0}^{\prime}\left(\theta^{*}(\tau)\right)\right\}\right] .
\end{aligned}
$$

From (53), as $\tau \rightarrow \infty$, we have

$$
\begin{aligned}
& \theta^{*}(\tau) \rightarrow 0, \quad \theta^{*}(\tau) \tau \rightarrow \infty, \quad \text { and } \\
& c_{0}-\phi_{0}\left(\theta^{*}(\tau)\right)+\theta^{*}(\tau) \phi_{0}^{\prime}\left(\theta^{*}(\tau)\right) \rightarrow c_{0}-\phi_{0}(0) .
\end{aligned}
$$

Hence $\lim _{\tau \rightarrow \infty} K(\tau)=-\infty$.

Remind that $K(\tau)$ is twice continuously differentiable. So, the lemma above shows that $K(\tau)$ takes a maximum at some point $\tau^{*} \in\left(\tau^{\dagger}, \infty\right)$, and at that point its derivative becomes zero, i.e. $K^{\prime}\left(\tau^{*}\right)=0$.

Lemma 6. The equation $K^{\prime}(\tau)=0$ has a unique solution $\tau=\tau^{*}$.

Proof. Under the assumption $\mathrm{C} 7$, we shall show that $K^{\prime \prime}\left(\tau^{*}\right)<0$ at any point $\tau^{*}$ at which $K^{\prime}\left(\tau^{*}\right)=0$.

We write the second order partial derivatives of $F(\theta ; \tau)$ by $F_{\theta \theta}(\theta ; \tau), F_{\theta \tau}(\theta ; \tau), F_{\tau \theta}(\theta ; \tau)$ and $F_{\tau \tau}(\theta ; \tau)$ as usual. In our case, clearly $F_{\theta \tau}(\theta ; \tau)=F_{\tau \theta}(\theta ; \tau)$.

Since $F_{\theta}\left(\theta^{*}(\tau) ; \tau\right)=\left.F_{\theta}(\theta ; \tau)\right|_{\theta=\theta^{*}(\tau)}=0$ from the definition of $\theta^{*}(\tau)$, by differentiating this equation on $\tau$ we have

$$
\begin{aligned}
0 & =\frac{d}{d \tau} F_{\theta}\left(\theta^{*}(\tau) ; \tau\right) \\
& =\left.\frac{\partial}{\partial \theta} F_{\theta}(\theta ; \tau)\right|_{\theta=\theta^{*}(\tau)} \cdot \frac{d \theta^{*}(\tau)}{d \tau}+\left.\frac{\partial}{\partial \tau} F_{\theta}(\theta ; \tau)\right|_{\theta=\theta^{*}(\tau)} \\
& =F_{\theta \theta}\left(\theta^{*}(\tau) ; \tau\right) \frac{d \theta^{*}(\tau)}{d \tau}+F_{\theta \tau}\left(\theta^{*}(\tau) ; \tau\right) .
\end{aligned}
$$

Hence

$$
\frac{d}{d \tau} \theta^{*}(\tau)=-\frac{F_{\tau \theta}\left(\theta^{*}(\tau) ; \tau\right)}{F_{\theta \theta}\left(\theta^{*}(\tau) ; \tau\right)} .
$$

On the other hand, the second derivative of $K(\tau)$ is given by

$$
\begin{aligned}
& K^{\prime \prime}(\tau)=\frac{d}{d \tau} F_{\tau}\left(\theta^{*}(\tau) ; \tau\right) \\
& =\left.\frac{\partial}{\partial \theta} F_{\tau}(\theta ; \tau)\right|_{\theta=\theta^{*}(\tau)} \cdot \frac{d}{d \tau} \theta^{*}(\tau)+\left.\frac{\partial}{\partial \tau} F_{\tau}(\theta ; \tau)\right|_{\theta=\theta^{*}(\tau)} \\
& =-F_{\theta \tau}\left(\theta^{*}(\tau) ; \tau\right) \frac{F_{\tau \theta}\left(\theta^{*}(\tau) ; \tau\right)}{F_{\theta \theta}\left(\theta^{*}(\tau) ; \tau\right)}+F_{\tau \tau}\left(\theta^{*}(\tau) ; \tau\right) \\
& =\frac{F_{\theta \theta}\left(\theta^{*}(\tau) ; \tau\right) F_{\tau \tau}\left(\theta^{*}(\tau) \tau\right)-\left\{F_{\theta \tau}\left(\theta^{*}(\tau) ; \tau\right)\right\}^{2}}{F_{\theta \theta}\left(\theta^{*}(\tau) ; \tau\right)}
\end{aligned}
$$

We calculate $K^{\prime \prime}(\tau)$ at $\tau=\tau^{*}$ where $\tau^{*}$ is a solution of $K^{\prime}(\tau)=0$. First we calculate the denominator of $(60)$.

$$
\begin{aligned}
& \text { [denominator of }(60)]=F_{\theta \theta}\left(\theta^{*}\left(\tau^{*}\right) ; \tau^{*}\right) \\
& =\tau^{*}\left\{2 \phi_{0}^{\prime}\left(\theta^{*}\left(\tau^{*}\right)+\theta^{*}\left(\tau^{*}\right) \phi_{0}^{\prime \prime}\left(\theta^{*}\left(\tau^{*}\right)\right)\right\}+\sigma_{0}^{2}\left\{\tau^{*}\right\}^{2 H_{0}}>0\right.
\end{aligned}
$$

Thus the denominator is positive. Next we calculate the numerator of $(60)$. 


$$
\begin{aligned}
& {[\text { numerator of }(60)] } \\
&=F_{\theta \theta}\left(\theta^{*}\left(\tau^{*}\right) ; \tau^{*}\right) F_{\tau \tau}\left(\theta^{*}\left(\tau^{*}\right) ; \tau^{*}\right)-\left\{F_{\theta \tau}\left(\theta^{*}\left(\tau^{*}\right) ; \tau^{*}\right)\right\}^{2} \\
&=\left\{\tau^{*}\left\{2 \phi_{0}^{\prime}\left(\theta^{*}\left(\tau^{*}\right)+\theta^{*}\left(\tau^{*}\right) \phi_{0}^{\prime \prime}\left(\theta^{*}\left(\tau^{*}\right)\right)\right\}+\sigma_{0}^{2}\left\{\tau^{*}\right\}^{2 H_{0}}\right\}\right. \\
& \times H_{0}\left(2 H_{0}-1\right) \sigma_{0}^{2}\left\{\theta^{*}\left(\tau^{*}\right)\right\}^{2}\left\{\tau^{*}\right\}^{2 H_{0}-2} \\
&-\left\{\theta^{*}\left(\tau^{*}\right) \phi_{0}^{\prime}\left(\theta^{*}\left(\tau^{*}\right)\right)+H_{0} \sigma_{0}^{2} \theta^{*}\left(\tau^{*}\right)\left\{\tau^{*}\right\}^{2 H_{0}-1}\right\}^{2} \\
&=-4 H_{0}\left(1-H_{0}\right) \phi_{0}^{\prime}\left(\theta^{*}\left(\tau^{*}\right)\right) \sigma_{0}^{2}\left\{\theta^{*}\left(\tau^{*}\right)\right\}^{2}\left\{\tau^{*}\right\}^{2 H_{0}-1} \\
&-H_{0}\left(1-H_{0}\right) \sigma_{0}^{4}\left\{\theta^{*}\left(\tau^{*}\right)\right\}^{2}\left\{\tau^{*}\right\}^{4 H_{0}-2} \\
&+H_{0}\left(2 H_{0}-1\right) \sigma_{0}^{2}\left\{\theta^{*}\left(\tau^{*}\right)\right\}^{3}\left\{\tau^{*}\right\}^{2 H_{0}-1} \phi_{0}^{\prime \prime}\left(\theta^{*}\left(\tau^{*}\right)\right) \\
&-\left\{\theta^{*}\left(\tau^{*}\right) \phi_{0}^{\prime}\left(\theta^{*}\left(\tau^{*}\right)\right)\right\}^{2} .
\end{aligned}
$$

The first two terms are negative. Since $K^{\prime}\left(\tau^{*}\right)=0$, from the second equation of (42) (or from (59)),

$$
\sigma_{0}^{2}\left\{\theta^{*}\left(\tau^{*}\right)\right\}^{2}\left\{\tau^{*}\right\}^{2 H_{0}-1}=\theta^{*}\left(\tau^{*}\right)\left\{c_{0}-\phi_{0}\left(\theta^{*}\left(\tau^{*}\right)\right)\right\} .
$$

So the last two terms of (62) are evaluated as

$$
\begin{aligned}
H_{0}\left(2 H_{0}-1\right) \sigma_{0}^{2}\left\{\theta^{*}\left(\tau^{*}\right)\right\}^{3}\left\{\tau^{*}\right\}^{2 H_{0}-1} \phi_{0}^{\prime \prime}\left(\theta^{*}\left(\tau^{*}\right)\right) & -\left\{\theta^{*}\left(\tau^{*}\right) \phi_{0}^{\prime}\left(\theta^{*}\left(\tau^{*}\right)\right)\right\}^{2} \\
\leq & \left\{\theta^{*}\left(\tau^{*}\right)\right\}^{2}\left\{c_{0}-\phi_{0}\left(\theta^{*}\left(\tau^{*}\right)\right)\right\} \phi_{0}^{\prime \prime}\left(\theta^{*}\left(\tau^{*}\right)\right) \\
& -\left\{\theta^{*}\left(\tau^{*}\right) \phi_{0}^{\prime}\left(\theta^{*}\left(\tau^{*}\right)\right)\right\}^{2} \\
= & -\left.\frac{1}{2}\left\{\theta^{*}\left(\tau^{*}\right)\right\}^{2} \frac{d^{2}}{d \theta^{2}}\left\{c_{0}-\phi_{0}(\theta)\right\}^{2}\right|_{\theta=\theta^{*}\left(\tau^{*}\right)} .
\end{aligned}
$$

We know that $\tau^{*}$ is in the interval $\left(\tau^{\dagger}, \infty\right)$ and hence $\theta^{*}\left(\tau^{*}\right)$ is in $\left(0, \theta^{\dagger}\right)$. From the assumption C7 (iii), $\frac{d^{2}}{d \theta^{2}}\left\{c_{0}-\phi_{0}(\theta)\right\}^{2}>$ 0 for $\theta \in\left(0, \theta^{\dagger}\right)$. Thus, (63) is negative, and the numerator of (60) is negative. This means that any point $\tau=\tau^{*}$ such that $K^{\prime}\left(\tau^{*}\right)=0$ is a maximal point of $F\left(\theta^{*}(\tau) ; \tau\right)$. Since there exist no solutions of $K^{\prime}(\tau)=0$ rather than maximal ones and the function $K(\tau)$ is continuously differentiable, the maximal point $\tau^{*}$ is unique.

Proof of Theorem 1. From Lemma 4, (41) is written as (42). Lemma 6 assures that the system of equations (42) has a unique solution $\left(\theta^{*}\left(\tau^{*}\right), \tau^{*}\right)$. Clearly the solution attains $\max _{\tau \in[0, \infty)} \inf _{\theta \in\left(0, \delta_{0}\right)} F(\theta ; \tau)$, and the max-inf value is greater than or equal to the one in (39) since the range of $\tau$ is enlarged. Thus (40) holds.

\section{CONCLUDING REMARKS}

We discussed the end-to-end backlog in a tandem network with mixed flows of tEBWs and fBms. The end-to-end back$\log$ can be evaluated by the backlog analysis of an imaginary single node model with the per-flow link capacity $c_{0}$ and the cgf of the per-flow input $\theta t \phi_{0}(\theta)+\sigma_{0}^{2} \theta^{2} t^{2 H_{0}} / 2$. However, the evaluation formula obtained is not so simple and needs some numerical calculations. In order to apply to an admission control, we should study further simplifications of the formula. Moreover, the formula may just provide a rough estimate of the bound, since in the process of aggregating multiple nodes into the single node, we set parameters and functions $c_{0}-\phi_{0}(\theta), \sigma_{0}^{2}$ and $H_{0}$ by using many inequalities. In particular, we anticipate that the formula would provide much overestimate, for example, in the case where one of the nodes, say node $i$, has enough link capacity and $\mathrm{fBm}$ cross flows with variance parameter $\sigma_{0}^{2}$ and Hurst parameter $H_{0}$, and another node, say node $i^{\prime}$, has only non$\mathrm{fBm}$ cross flows and the traffic load satisfies the equality $c_{i^{\prime}}-\phi_{\alpha}(\theta)-\phi_{\beta, i^{\prime}}(\theta)=c_{0}-\phi_{0}(\theta)$ in (32). The authors think that those issues are subjects of future work.

Finally the authors would like to thank the anonymous reviewers for their valuable comments.

\section{REFERENCES}

[1] C. S. Chang. Performance Guarantees in Communication Networks. Springer Verlag, 2000.

[2] F. Ciucu, A. Burchard and J. Liebeherr. A network service curve approach for the stochastic analysis of networks. In Proceedings of ACM Sigmetrics '05, 2005.

[3] M. Fidler and A. Rizk. A guide to the stochastic network calculus. IEEE Communication Surveys $\&$ Tutorials, 17(1):92-105, 2015.

[4] A. Ganesh, N. O'Connell and D. Wischik. Big Queues. Springer, 2004.

[5] Y. Jiang and Y. Liu. Stochastic Network Calculus. Springer, 2008.

[6] F. Kelly. Notes on effective bandwidths. In Stochastic Networks: Theory and Applications, Oxford University Press, 1996.

[7] K. Kobayashi and Y. Takahashi. Tractable effective bandwidths for end-to-end evaluation and fractional Brownian motion traffic. In Proceedings of Valuetools 2013, 2013.

[8] K. Kobayashi, Y. Takahashi and H. Takada. A Stochastic network calculus for many flow. In Proceedings of ITC21, 2009.

[9] K. Kobayashi, Y. Takahashi and H. Takada. A backlog evaluation formula for admission control based on the stochastic network calculus with many flows. IEICE Transactions on Communincations, E94-B(6):1288-1294, 2011.

[10] C. Li, A. Burchard and J. Liebeherr. A network calculus with effective bandwidth. Technical Report CS-2003-20, University of Virginia, Computer Science Department, 2003.

[11] J. Lieveherr, A. Burchard and F. Ciucu. Delay bounds in communication networks with heavy-tailed and self-similar traffic. IEEE Transactions on Information Theory, 58(2):1010-1024, 2012.

[12] ] Y. Liu, C.-K. Tham and Y. Jiang. A calculus for stochastic QoS analysis. Performance Evaluation, 64(6):547-572, 2007.

[13] I. Norros. A storage model with self-similar input. Queueing Systems, 16(3):387-396, 1994.

[14] A. Rizk and M. Fidler. Statistical end-to-end performance bounds for networks under long memory FBM cross traffic. In Proceedings of 18th IWQoS, 2010 . 